\title{
Lokales Übergangsmanagement weiterentwickeln
}

\author{
Dokumentation: Steuerungsgremium als Bedingung einer \\ gelingenden kommunalen Koordinierung der Jugendsozialarbeit
}

In der Bundesarbeitsgemeinschaft örtlich regionaler Träger der Jugendsozialarbeit (BAG ÖRT) sind rund 75 Träger der Jugendberufshilfe und Jugendsozialarbeit zusammengeschlossen. Die Organisation sieht ihre Aufgabe in der Unterstützung der Mitgliedseinrichtungen bei der sozialen und beruflichen Integration sozial benachteiligter und individuell beeinträchtigter junger Menschen. Dazu gehört auf Bundesebene die Weiterentwicklung des Aufgabenfeldes Jugendsozialarbeit in fachlicher und organisatorischer Hinsicht. Dabei beachtet und fördert der Verbund in besonderem Maße die Einbindung der Träger in örtlich-regionale, kommunale und wirtschaftliche Netzwerke. Internet http://www.bag-oert.de
Der Übergang von der Schule in Ausbildung und Beruf ist für viele junge Menschen langwierig und kompliziert geworden. Die Bundesarbeitsgemeinschaft örtlich regionaler Träger der Jugendsozialarbeit (BAG ÖRT) beschreibt in einem hier auszugsweise dokumentierten Positionspapier die Herausforderungen, vor denen das lokale Übergangsmanagement derzeit steht und formuliert notwendige Handlungsschritte und Entwicklungen für ein gelingendes Übergangsmanagement. Besonders wird dabei die Rolle der Träger der Jugendsozialarbeit beleuchtet.

Den direkten Zugang von der Schule in die Ausbildung schaffen nur ca. $60 \%$ der jungen Menschen. (1) Zwischen Schule und Arbeitswelt hat sich ein »Übergangssystem « mit zahlreichen unterschiedlichen Angeboten, die schwer zu überblicken sind, gebildet. Es existieren zahlreiche Projekte unterschiedlichster Akteure, die nicht oder nur unzureichend aufeinander abgestimmt und koordiniert sind. Diese Programme und Projekte werden kommunal, über die Länder oder den Bund finanziert und gesteuert.

Um mit diesen schwierigen Bedingungen umzugehen und die Angebote vor Ort sinnvoll zu steuern, wurde ein Ansatz entwickelt, der die Optimierung des Übergangssystems vor Ort zum Ziel hat. Das »Übergangsmanagement « hat bereits zahlreiche Ansätze in der Praxis und wird z. B. in der Förderinitiative "Regionales Übergangsmanagement « des Bundesministeriums für Bildung und Forschung (BMBF) als bundesweites Modellprogramm umgesetzt. Auch die Initiativen zur Entwicklung von kommunalen und lokalen Bildungslandschaften, wie zum Beispiel im Programm »Lernen vor Ort « des BMBF, haben zum Ziel, Bildungsangebote vor Ort zu koordinieren und $\mathrm{zu}$ steuern.

Die Träger der Jugendsozialarbeit sind wichtige Akteure im Übergangssystem. Sie gewährleisten eine intensive Beglei- tung für sozial benachteiligte und individuell beeinträchtigte Jugendliche an den Übergängen Schule-Ausbildung und Ausbildung-Beruf. Aus diesem Grund begrüßt und unterstützt die BAG ÖRT die bisherigen Bestrebungen, ein abgestimmtes Übergangsmanagement vor Ort zu installieren.

Aus unserer Sicht ist nun der entscheidende nächste Schritt, die Steuerungsverantwortung im Übergangsmanagement vor Ort systematisch zu verankern und ein verantwortliches Gremium zu bestimmen, das die Angebotsstruktur vor Ort koordiniert. Denn auch die Programme und Angebote des Übergangsmanagements, die gestalterischen Einfluss auf das Übergangssystem vor Ort nehmen, sind auf verschiedenen Ebenen angesiedelt, haben unterschiedliche Zielgruppen, agieren zum Teil parallel und sind mit unterschiedlichen Handlungsspielräumen ausgestattet.

In besonderer Verantwortung bei der Gestaltung des Übergangs Schule-Beruf sind die Kommunen. Auch wenn es keine formale rechtliche Zuständigkeit der Kommunen gibt, sehen wir die Verantwortung für die berufliche und soziale Integration von jungen Menschen hier verortet, denn die Kommune ist »der Ort, an dem schulisches, soziales und emotionales Lernen und Bilden stattfindet " und ist damit »die zentrale Plattform für die Bildung junger Menschen «. (2) Die Steuerungsverantwortung für die Koordinierung der Akteure und Angebote muss in der Kommune wahrgenommen werden. Daher fordern wir die Umsetzung einer Kommunaler Koordinierung und ergänzen mit unserer Position die Forderungen der Weinheimer Initiative. (3)

Die erfolgreiche Kommunale Koordinierung ist ein besonders wichtiger Schritt auf dem Weg zu einem qualitativ hochwertigen Übergangsmanagement. Aus diesem Verständnis heraus müssen die Kommunen im Übergangssystem agieren. Sie müssen koordinierende Gremien un- 
ter Beteiligung der wichtigsten Finanzierungs- und Angebotsakteure initiieren und die Leitung übernehmen. Dabei soll die lokale politische Spitze die Verantwortung für die Kommunale Koordinierung übernehmen und den Leiter bzw. die Leiterin des Steuerungsgremiums bestimmen. Der Leiter bzw. die Leiterin sollte Entscheidungsträger/in in der Kommune sein. Im Steuerungsgremium müssen alle Akteure im Übergangssystem eingebunden sein. Dazu gehören selbstverständlich auch die Träger der Jugendsozialarbeit sowie alle bereits vorhandenen Koordinierungsakteure.

Um das Übergangsmanagement vor Ort durch eine Kommunale Koordinierung erfolgreich zu gestalten, sind aus Sicht der BAG ÖRT folgende Bedingungen zu beachten:

- Die jungen Menschen stehen im Mittelpunkt: Alle beteiligten Akteure vor Ort müssen trotz unterschiedlicher Rechtsgrundlagen, Förderlogiken, Zuständigkeiten und Teilziele immer die betroffenen jungen Menschen im Blick haben und behalten. Interessendifferenzen durch verschiedene Zuständigkeiten und Ziele müssen harmonisiert werden und die Akteure sind in der Pflicht immer wieder über die Angebotsstruktur einen Konsens im besten Interesse der jungen Menschen zu finden.

- Träger der Jugendsozialarbeit als wichtige Partner und Akteure im Übergangssystem: Die Träger der Jugendsozialarbeit sind wichtige Akteure im Übergangssystem. Sie vertreten die Gruppe der sozial benachteiligten und individuell beeinträchtigten jungen Menschen, die in erhöhtem Maß auf einen gut funktionierenden Übergang angewiesen sind. Die Träger der Jugendsozialarbeit besitzen besondere Kenntnisse der Bedarfe ihrer Zielgruppen, aber auch besondere Kenntnisse der Strukturen und Angebote vor Ort. Sie können neue Programme und Angebote auf ihren regionalen Nutzen einschätzen und bewerten. Dies gilt vor allem für Träger mit besonderem regionalem und kommunalem Bezug. Sie sind als Experten in die Kommunale Koordinierung einzubinden.

- Steuerungsgremium unter Leitung der kommunalen Spitze: Kommunale Koordinierung kann nur dann gelingen, wenn die Kommune Verantwortung übernimmt. Das Gremium, das für die
Steuerung der Angebote vor Ort zuständig ist, steht unter kommunaler Leitung und besteht aus Vertretern und Vertreterinnen aller wichtigen Akteure des Übergangsmanagements. Dieses Gremium gewährleistet eine ausreichende Kommunikation zwischen den Akteuren im Übergangssystem und garantiert eine neutrale Steuerung im Interesse der jungen Menschen vor Ort.
Weise einbezogen werden. Dies ersetzt natürlich nicht die Notwendigkeit und Pflicht der Ministerien auf Bundes- und Landesebene, Programme im Bereich Übergang Schule-Arbeitswelt aufeinander abzustimmen. In besonderer Verpflichtung zur Absprache und Abstimmung sind die Vertreter/innen der Rechtskreise der Sozialgesetzbücher (SGB) II, III und VIII, um bedarfsge-

\section{"Die Kommune ist der Ort, an dem schulisches, soziales und emotionales Lernen stattfindet «}

- Mitglieder des Steuerungsgremiums: In das Steuerungsgremium sollen alle Akteure eingebunden werden, die am Übergang Schule-Arbeitswelt arbeiten. Die genaue Struktur des Gremiums hängt von den Gegebenheiten vor Ort ab. Die Mitglieder des Steuerungsgremiums sollen Entscheidungsträger/innen in ihren Organisationen sein, um verbindliche Absprachen und Entscheidungen im Steuerungsgremium treffen zu können. Einbezogen werden sollen:

- Kommunale Spitze, kommunale Vertreter/innen,

- Träger der Jugendsozialarbeit,

- Agentur für Arbeit,

- Örtliche Grundsicherungsträger,

- Öffentliche und Freie Jugendhilfe,

- Schule,

- Wirtschaft,

- Initiativen und Programme, die Übergangsgestaltung bzw. Übergangsmanagement zum Ziel haben, wie z. B. das Programm »Regionales Übergangsmanagement ", das Programm »Kompetenzagenturen « und »Lernen vor Ort «.

Zur engen Einbindung möglichst vieler Akteure wäre außerdem die Etablierung eines Fachbeirats neben dem Steuerungskreis denkbar, der selbst keine steuernde Funktion hat, den Steuerungskreis aber regelmäßig berät.

- Aufgaben des Steuerungsgremiums: Bei der Abstimmung von Bundes- und Landesprogrammen und Angeboten auf kommunaler Ebene muss das Koordinierungsgremium in geeigneter recht Angebote zu planen und Parallelstrukturen zu vermeiden. Das Steuerungsgremium ist für eine Ist-Analyse der Situation vor Ort und eine umfassende Bedarfsplanung verantwortlich und gibt Empfehlungen an Entscheidungsgremien und Entscheidungsträger $\mathrm{ab}$, um eine Abstimmung zwischen verschiedenen Programmen und Projekten herzustellen und Parallelstrukturen zu vermeiden. Das Steuerungsgremium ist außerdem die wichtigste Informationsund Austauschplattform für die Akteure am Übergang Schule- Leitung durch die kommunale Spitze Arbeitswelt. Hier wird über neue Projekte und Programme informiert und zukünftige Bedarfe werden erhoben sowie passende Angebote initiiert. Das Gremium ist auch dafür verantwortlich, Qualitätsstandards für das Übergangsmanagement zu erarbeiten und diese regelmäßig zu überprüfen. Das Steuerungsgremium legt regelmäßig Ziele für das Übergangsmanagement fest, überprüft und evaluiert die Ziele und den Zielerreichungsgrad. Die Akteure des Steuerungsgremiums verpflichten sich, die festgelegten Ziele zu verfolgen und umzusetzen.

- Konstante, flächendeckende Strukturen und die Verstetigung von Kommunaler Koordinierung: Auf der Grundlage einer entsprechenden politischen Beschlussfassung muss das Steuerungsgremium dauerhaft eingerichtet sein und regelmäßig unter kommunaler Leitung tagen. Die getroffenen Absprachen sind 
verbindlich und damit Handlungsmaxime. Kommunale Koordinierung des Übergangsmanagements darf nicht auf Modellregionen beschränkt bleiben, sondern soll die Angebote für junge Menschen in ganz Deutschland steuern und optimieren.

Berlin, im Dezember 2009

Kontakt: Bundesarbeitsgemeinschaft örtlich regionaler Träger der Jugendsozialarbeit (BAG ÖRT), Marienburger Straße 1, 10405 Berlin, Telefon 030 40505769-0, Fax 030 40505769-19, E-Mail info@bagoert.de, Internet http://www.bag-oert.de

\section{Anmerkungen}

(1) Autorengruppe Bildungsberichterstattung (Hg.) (2008): Bildung in Deutschland 2008. Ein indikatorgestützter Bericht mit einer Analyse zu Übergängen im Anschluss an den Sekundarbereich I, S. 96.

(2) Deutscher Verein (2007): Diskussionspapier des Deutschen Vereins zum Aufbau Kommunaler Bildungslandschaften, S.2, abrufbar unter http://www.deutscher-verein.de/05empfehlgen/empfehlungen2007/pdf/Di skussionspapier_des_Deutschen_Vereins_zum_Aufbau_Kommunaler_Bildungslandschaften.pdf.

(3) Die Weinheimer Initiative schlägt in einer Erklärung 2007 »Lokale Verantwortungsgemeinschaften « und eine "Kommunale Koordinierung « vor. Dabei wird Kommunale Koordinierung als »Kommunal verantwortete Bündelung und Abstimmung von Aktivitäten, die Herstellung von Transparenz und die Entwicklung und Anwendung gemeinsam vereinbarter Qualitätsstandards für den Bereich des Übergangs von der Schule in Beruf und Arbeitswelt " verstanden. (Weinheimer Initiative [2007] [Hg.]: Lokale Verantwortung für Bildung und Ausbildung. Eine öffentliche Erklärung, S. 7). An diese Erklärung schließt dieses Papier an.

\section{Neue Wege wagen}

Probleme und Lösungsmöglichkeiten der Innovationspolitik des Bundes

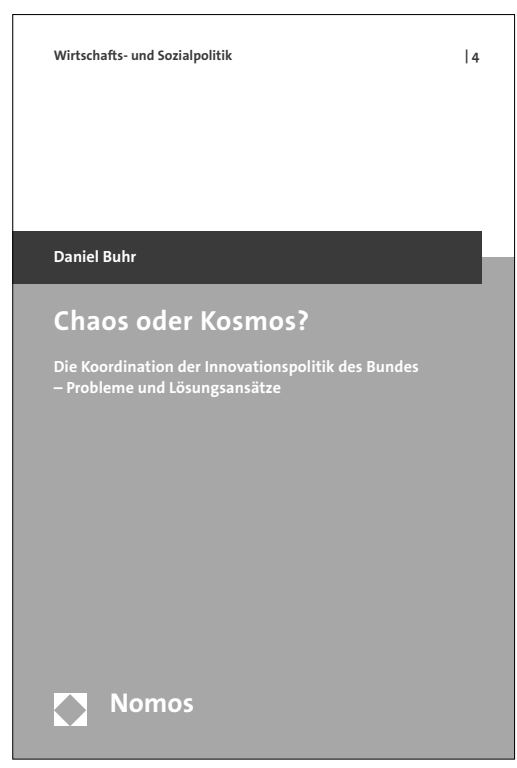

\author{
Chaos oder Kosmos? \\ Die Koordination der Inno- \\ vationspolitik des Bundes - \\ Probleme und Lösungsansätze \\ Von Dr. Daniel Buhr \\ 2010, 266 S., brosch., 39,-€, \\ ISBN 978-3-8329-5504-5 \\ (Wirtschafts- und Sozialpolitik, \\ Bd. 4)
}

Wie entstehen Innovationen und welche Rolle spielt dabei die Innovationspolitik? Wie wird sie koordiniert und was ließe sich verbessern? Das vorliegende Buch beantwortet diese Fragen. Der Autor wählt dabei bewusst einen interdisziplinären Zugriff und richtet sich an Leserinnen und Leser aus Wissenschaft und Praxis - in der Politik, Verwaltung und Wirtschaft.

Unter Koordination verstehen wir gemeinhin das Abstimmen verschiedener Aktivitäten aufeinander. Dieses Abstimmen tritt im vorliegenden Kontext in unterschiedlichen Variationen auf - die „Koordinierungstrias“ erstreckt sich vom Wirtschaftssystem (koordinierter Kapitalismus) über das politische System (koordiniertes politisch-administratives System) bis hin zur Innovationspolitik als Querschnittsfeld. Deren Betätigungsfeld ist dementsprechend groß und die Akteure sind vielfältig - auf kommunaler und regionaler, auf Länder- und Bundesebene sowie auf europäischer Ebene. Bei der Steuerung dieses komplexen Politikfeldes scheinen die bestehenden Institutionen an ihre Grenzen zu stoßen. Das Buch benennt die Herausforderungen und zeigt Lösungswege auf.

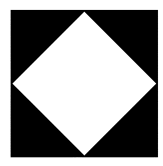

\section{Nomos}

Bitte bestellen Sie im Buchhandel oder

versandkostenfrei unter $\bullet$ www.nomos-shop.de 